\title{
Systematic review of pancreaticoduodenectomy for locally advanced gastric cancer
}

\author{
Patrick Roberts $\cdot$ Rajini Seevaratnam $\cdot$ \\ Roberta Cardoso - Calvin Law $\cdot$ Lucy Helyer • \\ Natalie Coburn
}

Received: 3 May 2011/Accepted: 29 July 2011/Published online: 26 August 2011

(C) The International Gastric Cancer Association and The Japanese Gastric Cancer Association 2011

\begin{abstract}
Background The purpose of this study was to identify and synthesize findings from all articles on surgical and longterm outcomes in patients with gastric cancer undergoing gastrectomy combined with pancreaticoduodenectomy (PD).

Methods Electronic literature searches were conducted using Medline, EMBASE, and Cochrane databases from January 1, 1985, to December 31, 2009.

Results Eight retrospective case series were included, with 132 patients having PD combined with gastrectomy. PD was combined with total gastrectomy in 27 patients, and subtotal gastrectomy in 81 patients; 24 patients had
\end{abstract}

Electronic supplementary material The online version of this article (doi:10.1007/s10120-011-0086-5) contains supplementary material, which is available to authorized users.

\section{P. Roberts}

Division of General Surgery, University of Toronto,

Toronto, Canada

P. Roberts $\cdot$ C. Law $\cdot$ N. Coburn

Division of Surgery, Sunnybrook Health Sciences Centre,

University of Toronto, Toronto, Canada

R. Seevaratnam $\cdot$ R. Cardoso $\cdot$ N. Coburn

Sunnybrook Research Institute, Sunnybrook Health

Sciences Centre, Toronto, Canada

L. Helyer

Division of General Surgery, Department of Surgery,

Dalhousie University, Halifax, Canada

N. Coburn $(\square)$

Division of Surgical Oncology, Odette Cancer Centre, Sunnybrook Health Sciences Centre, 2075 Bayview Ave, Suite T2-60, Toronto, ON M4N 3M5, Canada

e-mail: natalie.coburn@sunnybrook.ca undocumented gastric resection type. Clinical stage was available for 92 patients (4 stage I, 7 stage II, 26 stage III, and 55 stage IV). Five studies (98 patients having PD combined with gastrectomy) compared PD and gastrectomy to gastrectomy alone. In the four studies reporting morbidity, PD had a higher morbidity. The pooled pancreatic anastomotic leak rate was $24.5 \%$ for the seven studies in which complications were reported; however, there were no peri-operative deaths. Long-term survival (37.3\% at 5 years) in gastric cancer patients with PD combined with gastrectomy was described; however, survival was poor in the setting of incurable factors. Due to heterogeneity of patients and staging techniques in the case series no recommendations can be made on the appropriate selection criteria for patients undergoing PD and gastrectomy.

Conclusion PD for gastric cancer invading the pancreas is associated with a higher morbidity; given the heterogeneous data, defining exact selection criteria is difficult.

Keywords Gastric cancer - Pancreaticoduodenectomy · Gastrectomy

\section{Introduction}

In North America and Europe, gastric cancer commonly presents at a late stage with frequent involvement of surrounding organs, and is generally associated with poor outcomes [1,2]. The pancreas is involved in up to $50 \%$ of advanced cases; often it is the only organ involved [3, 4].

An R0 resection of gastric cancer is essential to achieve long-term survival, and at times this can only be obtained by multivisceral resection [5, 6]. Several studies have reported that combined resection of adjacent organs such as 
spleen, colon, or distal pancreas is associated with improved survival in patients with locally invasive gastric cancer [5, 7, 8]. In cases of invasion of the head of the pancreas or extensive infiltration into the duodenum, a pancreaticoduodenectomy (PD) would be necessary to achieve cure (R0) and improve overall survival (OS). However, given the risk of complications associated with PD, and unknown benefit in terms of OS, a palliative bypass has often been advocated as the procedure of choice [4].

Advances in peri-operative management and surgical technique have resulted in a lower morbidity and mortality associated with a PD; consequently, the indications for this procedure have been expanded. Favorable results of PD for locally advanced gastric cancer have been reported in a few studies, but it remains a controversial procedure [4, 9-15]. The purpose of this study was to identify and synthesize short- and long-term outcomes from all articles on patients undergoing gastrectomy combined with PD for gastric cancer.

\section{Methods}

\section{Data sources}

Electronic literature searches were conducted in Medline and EMBASE from January 1, 1985, to December 31, 2009, according to the search algorithm presented in Appendix A. Search terms included: [exp Stomach Cancer/ or (((gastric or stomach) adj1 cancer\$) or ((gastric or stomach) adj1 carcinoma) or ((gastric or stomach) adj1 adenocarcinoma) or ((gastric or stomach) adj1 neoplasm\$)).mp.] and [((negative or resection) adj2 margin\$).mp. or exp frozen section/or exp GASTRECTOMY/ or ((gastric or stomach) adj2 resect\$).mp. [mp $=$ title, abstract, subject headings, heading word, drug trade name, original title, device manufacturer, drug manufacturer name] or omentectom\$.mp. or multivisceral resection\$.mp.] and [clinical trial/or controlled clinical trial/or exp comparative study/or meta analysis/or multicenter study/or exp practice guideline/or randomized controlled trial] not [Case Report/or review]. A separate search of the Cochrane Central Register of Controlled Trials (1985-2009) was performed using the search term "gastric cancer". No attempt was made to locate unpublished material.

Study selection and review process

To be eligible, studies had to meet the following criteria: (1) PD in patients diagnosed with histopathology-confirmed gastric adenocarcinoma; (2) complication or survival data was reported; (3) published in peer-reviewed journals from 1985 to 2009; and (4) published in English. Studies were excluded according to the following exclusion criteria: (1) involved animals and/or ex vivo samples; (2) involved patients with mixed cancer or studies investigating PD in other cancers with no separate analysis of gastric cancer subjects; (3) studies that did not provide sufficient information to determine complication or survival data; (4) review articles, meta-analyses, abstracts, conference proceedings, and editorials/letters; and (5) duplicated case reportings. All electronic search titles, selected abstracts, and full-text articles were independently reviewed by a minimum of two reviewers (NC, LH, RC, and RS). Reference lists from review papers and relevant articles were also examined for additional studies that met our inclusion criteria. Disagreements on study inclusion/ exclusion were resolved with a consensus meeting.

\section{Data extraction}

A systematic approach to data extraction was used to produce a descriptive summary of participants, interventions, and study findings. The first reviewer (RS) independently extracted the data and a second reviewer (PR) checked the data extraction. No attempt was made to contact authors for additional information.

\section{Results}

A total of 142 titles/abstracts were identified from the electronic searches and reference lists as multivisceral resection articles with possible PD cases for preliminary review. After removal of duplicates and screening for relevant titles, abstracts, and reference lists, a total of 11 articles were submitted for a full-text review. There were no prospective or randomized series (Table 1); consequently, 8 retrospective case series [4,9-11] were included in this review (Fig. 1).

One hundred and thirty-two patients were identified as having PD combined with gastrectomy; 68 were male, 42 female, and gender was unavailable for 22 patients. PD was combined with total gastrectomy in 27 patients, with subtotal gastrectomy in 81 patients, and the gastric resection type was not documented in 24 patients. The clinical stage was available for 92 patients: 4 were stage I; 7 stage II; 26 stage III; and 55 stage IV. T stage was available for 115 patients (37 T1-2, $19 \mathrm{~T} 3$, and $59 \mathrm{~T} 4)$. The nodal stage was available for 112 patients (24 N0; $17 \mathrm{~N} 1 ; 29 \mathrm{~N} 2 ; 42 \mathrm{~N} 3$ ); 10 patients were described as node-positive, but a definitive nodal stage was not given, and 10 patients were described as N1-2. All patients were staged using the Japanese Research Society for Gastric Cancer (JRSGC) 


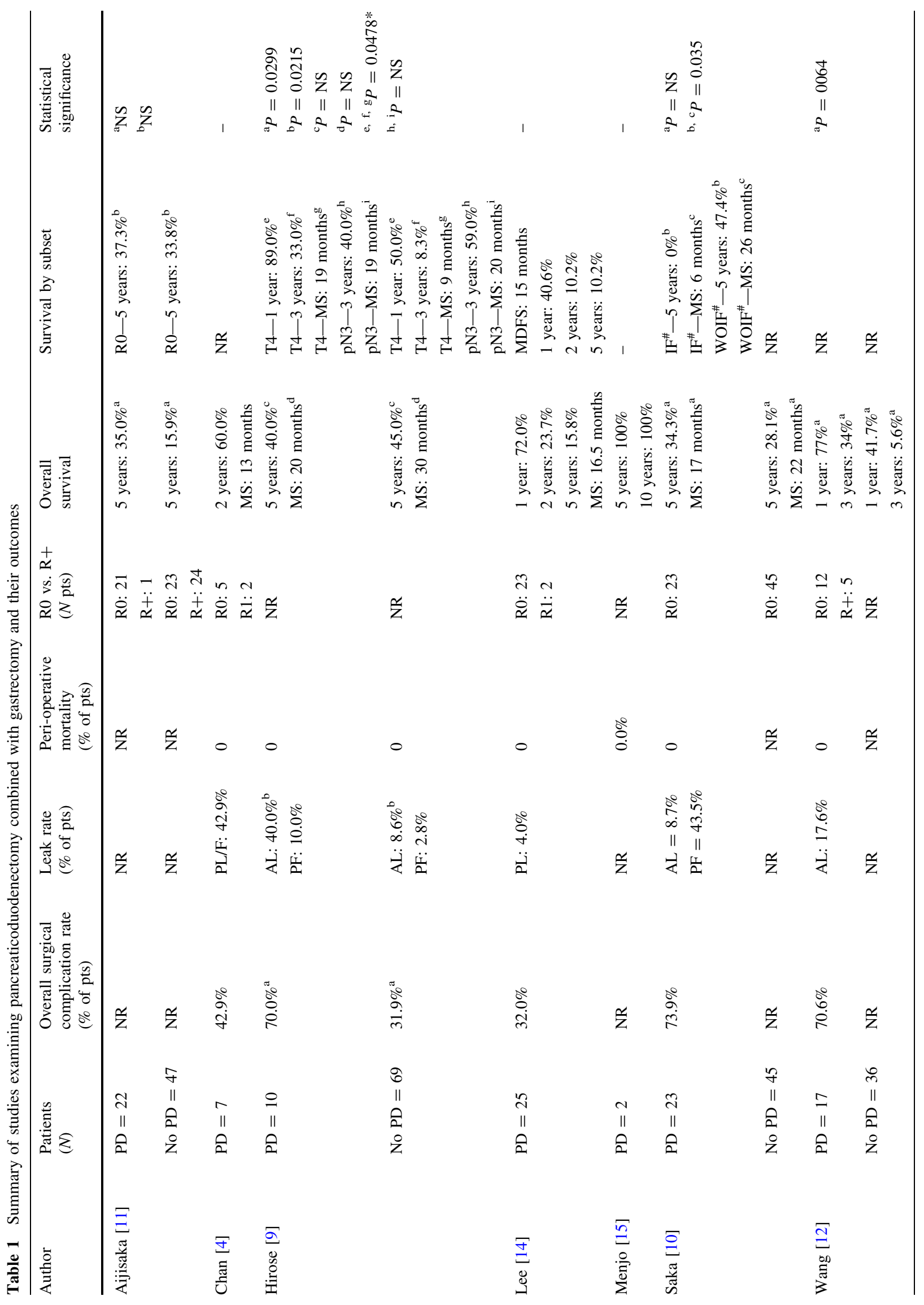




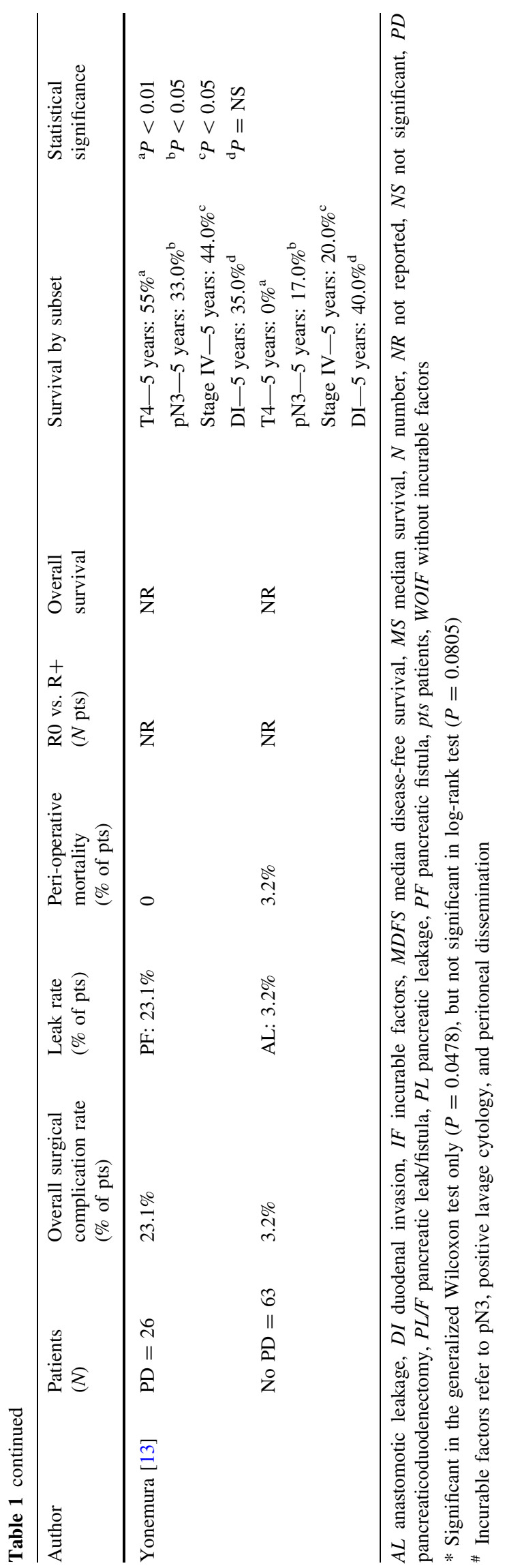

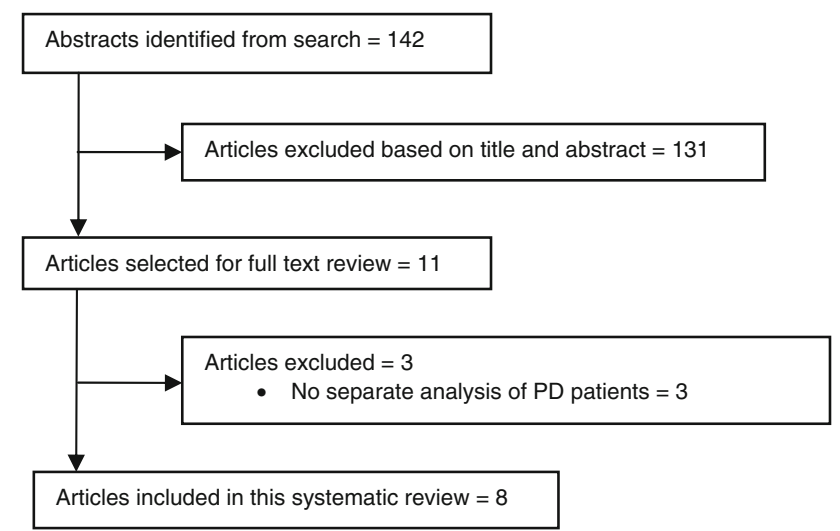

Fig. 1 Article selection flow. $P D$ pancreaticoduodenectomy

classification system. Five of the 42 patients with N3 disease were staged using the 2nd English edition of the JRSGC staging.

The indications for PD and gastrectomy were duodenal invasion beyond the pyloric ring by gastric cancer, gastric cancer infiltration into the pancreas, and nodal metastases around the pancreatic head. The timing of the decision to perform a PD with gastrectomy was provided in 2 out of the 8 studies; in one study the decision to perform PD was described as based on pre-operative staging [14], while in another it was based on operative findings [4, 9-13, 15]. The utilization of intra-operative frozen sections to determine margin status prior to embarking upon a PD was not noted.

Six of the eight studies did not describe or report on staging investigations used prior to PD and gastrectomy [913, 15]. Chan reported performing endoscopic ultrasound in 4 of 7 patients; pancreatic invasion was detected in one patient and was confirmed histologically [4]. The use of computed tomography (CT) or magnetic resonance imaging (MRI) was not reported in Chan's study. Lee reported staging investigations with abdominal CT scan, and if needed, chest $\mathrm{CT}$ and/or bone scan to rule out metastasis; PD was then performed after the exclusion of metastatic disease [14].

Four of the eight studies reviewed did not report the usage of chemotherapy or radiation therapy $[10,11,14$, 15]. Neoadjuvant chemotherapy was described in one study and this was used in four of 7 patients only [4]. Adjuvant chemotherapy was used in four of the eight studies, with one study not reporting the number of patients receiving adjuvant chemotherapy $[4,9,12,13]$. The number of patients known to have received adjuvant chemotherapy was 52. The chemotherapeutic regimens reported for adjuvant therapy were mitomycin $\mathrm{C}$ with 5 -fluorouracil; etoposide, leucovorin, and 5-fluorouracil; and mitomycin C with tegafur $[9,12,13]$. Radiation therapy was not reported in any of the studies reviewed. 
In all studies, patients undergoing PD had a higher morbidity. The morbidity associated with gastrectomy and PD was higher than that associated with gastrectomy alone (23.1-73.9\% compared to $3.2-31.9 \%$ ) [9, 10, 13]. The pooled pancreatic leak rate was $24.5 \%$ for the five studies in which it was reported; there were no peri-operative deaths.

Five studies (98 patients having PD combined with gastrectomy), compared survival outcomes of PD and gastrectomy to those with gastrectomy alone [9-13] (Table 1). In their study of patients with extension into the duodenum, Ajisaka et al. [11] reported no difference in 5-year OS between the group with PD and gastrectomy versus those undergoing gastric resection alone for duodenal invasion by gastric cancer once a negative margin was achieved (37.3 and $33.8 \%$, respectively). However, they showed a trend towards worse survival in the gastrectomy-alone patients compared to the PD patients when the gastrectomy group included both patients with positive and negative resection margins (5-year OS of 15.9 vs. 35\%; not significant [NS]). Comparing patients who underwent a PD combined with gastrectomy with those who underwent gastrectomy alone, Hirose et al. [9] found median survival was improved for patients with T4 disease (19 vs. 9 months, $P=0.0478$ ), but not for those with N3 disease (19 vs. 20 months, NS). Saka et al. [10] showed that PD combined with gastrectomy achieved a 5-year OS of $34.3 \%$. Patients without incurable factors (para-aortic lymph node metastasis, positive lavage cytology, and peritoneal dissemination) had a 5-year OS of $47.4 \%$, while those with incurable factors undergoing PD and gastrectomy had a 5-year OS rate and median survival of $0 \%$ and 6 months, respectively [10]. Wang et al. [12] analyzed patients with disease in the pancreatico-duodenal region and reported a significant difference $(P=0.0064)$ in survival for those undergoing a PD with gastrectomy $(34 \%$ 3 years OS) compared to a palliative and explorative group of patients with gastric cancer (5.6\% 3-year OS). The latter group was composed of a heterogeneous set of patients who underwent a palliative gastrectomy, bypass, or exploration only, or no resection due to the presence of additional metastases or poor physical state [12]. Yonemura et al. [13] examined the survival of 89 patients with distal gastric cancer with duodenal invasion, invasion to the pancreatic head, or N3 metastases; 26 of these patients underwent PD, gastrectomy, and right colectomy, while 63 underwent a gastric resection only. Survival was better in the multivisceral group compared to the simple gastrectomy group for those patients with infiltration of cancer into the head of the pancreas, N3 metastases, and/or stage IV disease, but not for those with duodenal invasion [13].

Within the series of 132 cases, at least 19 patients (14\%) who had PD combined with gastrectomy survived beyond
5 years. However, this maybe an underestimate of the actual achievable survival, as some publications did not report on individual patient survival. The stage was available for 11 of these 19 patients surviving beyond 5 years $(2$ stage I, 6 stage III, and 3 stage IV by the Japanese clinical staging system). Four patients survived for 10 years (1 stage II, 1 stage III, and 2 stage IV). In Yonemura's study, the 5-year overall survival for stage IV patients (by the JRSGC staging system) was $44 \%$ after PD combined with gastrectomy [13].

\section{Discussion}

Gastric cancers with invasion of adjacent organs portend a poor prognosis for patients and present a formidable surgical challenge. The principles of curative-intent surgical oncology demand an en-bloc resection of organs potentially invaded by cancer. In cases of duodenal or pancreatic invasion, this necessitates a PD with a gastrectomy. For many years, the operative risk of the PD outweighed the potential benefit, but with advances in surgery and improvements in peri-operative care, it has become a more viable option to attain long-term survival.

Prediction of the overall survival of patients with gastric cancer and selection of the most beneficial treatment plan/ operative strategy depends on accurate staging. However, this is problematic. Some patients may have microscopic disease not recognized at the time of the operation, while others may be, inaccurately, presumed to have tumor invasion into contiguous organs due to large inflammatory perigastric lymph nodes or extensive desmoplastic reaction surrounding the tumor. This difficulty in T-stage assessment may result in a patient mistakenly undergoing an extended gastric resection or being deemed palliative. Indeed, over-staging was documented in $34 \%$ of patients in the Ozer series, $36.6 \%$ of the Jeong series, and $33 \%$ of the Hirose series [6, 9, 16]. Over-staging may have led to patients undergoing an unnecessary multivisceral resection, when a simple gastrectomy would have resulted in the same long-term outcome. Unfortunately, the accuracy for determining T4 status by CT $(80 \%$ with the use of multidetector CT scans [17]), or endoscopic ultrasound (79\%, [18]) is far from perfect. The pre-operative radiologic investigations that occurred prior to these resections were not described by the majority of the primary articles and only one study reported the use of preoperative endoscopic ultrasound (EUS). Increasing experience with EUS may greatly benefit patients with advanced gastric cancer, by decreasing the number of patients who are over-staged, thus preventing a multivisceral resection, with its associated complications, in patients who do not have true adjacent organ invasion. Unfortunately, experienced 
clinicians recognize the difficulty in determination of adjacent organ invasion and they also recognize that the decision for multivisceral resection must often be based on surgical findings [16].

Morbidity rates were not satisfactorily reported in some of the papers included in this review, leading to an inability to pool the morbidity data. However, from the morbidity data that were reported, the complication rate associated with gastrectomy and PD was higher than that associated with gastrectomy alone. The main complication unique to the PD group was pancreatic duct leakage, with a pooled average rate of $24.5 \%$, which is similar to the $26.7 \%$ reported pancreatic leak rate in the pancreatic cancer literature [19]. There are multiple definitions and grading systems for pancreatic leaks, and reported rates of leak will vary depending upon which definition is used [19]. The pancreatic leaks in this analysis were managed mainly conservatively and there were no peri-operative mortalities, meaning most were low grade, or grade A according to the International Study Group on Pancreatic Fistula [20].

A recently published study showed that the 5-year survival rate of gastric cancer invading adjacent organs was influenced by histologic type, lymph node metastasis, liver metastasis, peritoneal dissemination, extent of lymph node dissection, and curability of operation [21]. Of these, independent prognostic factors for survival were lymph node metastasis, classified according to the Union International Contre le Cancer (UICC)/American Joint Committee on Cancer (AJCC) 6th edition guidelines (N2, N3 versus N0, N1, relative risk 2.028, $P<0.001$ ), liver metastasis (present versus absent, relative risk 1.582, $P=0.023$ ), and curative resection (no versus yes, relative risk 1.719, $P<0.001)$. A significant survival benefit for curative resection (R0) was observed, with a 5-year OS rate of $21.5 \%$ compared with non-curatively resected cases (R1) $(5.1 \%)$ [21]. These factors all appear to be important in the selection of patients for PD combined with gastrectomy; however, the exact criteria for selecting patients for PD cannot be fully elucidated in the data found in the 8 articles examined in this review.

Appropriate patient selection is critical for achieving long-term survival. Saka et al. [10] showed that patients who underwent a PD with gastrectomy, in the absence of incurable factors (para-aortic lymph node metastasis, positive lavage cytology, and peritoneal dissemination) had a median survival of 26 months and achieved a $47.4 \%$ 5 -year survival in comparison to a median survival of 6 months in those patients with incurable factors. Clearly, prior to embarking upon this type of multivisceral resection, thorough preoperative investigations must be performed, including peritoneal lavage and CT imaging to rule out metastatic disease. However, in the majority of the 8 articles in the present review, pre-operative investigations are not described and thus the most efficacious staging method cannot be elucidated.

An R0 resection must be achieved, as long-term survivors with positive margin resections or residual disease are rare; Saka et al. [10] found a 7.9\% 5-year survival in R1 patients. Similarly, Wang et al. [12] also found significantly worse survival outcomes for patients with positive microscopic margins following PD combined with gastrectomy $(P=0.0174)$. When negative margins were achieved, Ajisaka et al. [11] showed the survival outcome of PD combined with gastrectomy to be equal to that of simple gastrectomy (37.3 and 33.8\%, respectively, NS). Wang proposed PD in combination with gastrectomy for: (1) head of pancreas invasion by gastric cancer, (2) infiltration of gastric cancer into the duodenum, and (3) invasion of the inferior segment of the common bile duct by gastric cancer [12]. Although it is difficult to draw conclusions based upon the small number of patients in the present systematic review, the benefit of PD combined with gastrectomy appears to be most pronounced in patients with direct extension of a gastric cancer into the pancreas, as illustrated by the T4 cancers of the Yonemura series, where a highly significant difference in survival was found between patients undergoing a PD combined with gastrectomy compared to simple gastrectomy for invasion of the pancreas [13]. Alternatively, there were mixed outcomes for patients resected with a PD combined with gastrectomy when the indication for PD was infiltration into the duodenum. Yonemura et al. found no difference in survival for patients who underwent a PD or gastrectomy for duodenal invasion [13]. Ajisaka et al. [11] examined 69 cases of mucosal, submucosal, and nodal invasion into the duodenum. In the patients resected curatively, there was improved 5-year OS for mucosal involvement (45.3\%) compared to results for submucosal (13.3\%) and nodal $(0 \%)$ involvement. The type of duodenal invasion likely represents the biologic aggressiveness of the cancer and thus must be considered before embarking upon an extended resection. For both direct invasion into the pancreas and duodenal infiltration, there may have been overstaging, and thus multivisceral resection occurred in patients who did not benefit from this aggressive resection. For example, although Saka et al. [10] report an overall 5-year survival of $47.4 \%$ for patients who did not have incurable factors, of the nine patients who did not die from gastric cancer, four had T2 cancers, 2 had T3 cancers, and only 3 had T4 cancers. Ajisaka et al. [11] report that the average length of duodenal invasion was $17.7 \pm 6.6 \mathrm{~mm}$, which, in many cases, may be resected with an extended duodenal resection, rather than a formal PD. In fact, they show that the survival for patients with $\geq 30 \mathrm{~mm}$ compared to $<30 \mathrm{~mm}$ was not significantly different, although the margin status for these cases is not clear. 
The most controversial indication for PD combined with gastrectomy is that of peri-pancreatic lymph node involvement. Ajisaka et al. [11] found no 2-year survivors for patients with nodal invasion into the duodenum, regardless of the resection technique. Likewise, Hirose found no difference in median survival (19 vs. 20 months, NS) between the PD and simple gastrectomy groups in patients with N3 disease [9]. Contradicting these findings, Yonemura et al. [13] did find a statistically significant difference in survival for patients with Japanese Staging System N3 disease who underwent PD combined with gastrectomy and right colectomy compared to those with gastrectomy alone. The addition of the right hemi-colectomy was rationalized as necessary for nodal clearance, and may be partially responsible for this difference in results.

It is important to note that although all of the included articles in this review utilized the Japanese T, N, M classification system, some used the 1st English edition published by the JRSGC in 1995 [22], some used JRSGC editions previously published in Japanese, while others used the 2nd English edition published by the Japanese Gastric Cancer Association (JGCA) in 1998 [23]. The main difference between the 1st and 2nd English editions is the classification of $\mathrm{N}$ stage. In the 1st English edition there were five $\mathrm{N}$ stages (N0, N1, N2, N3, N4), with N3 referring to metastases in the hepatoduodenal, pre- and retropancreatic, and superior mesenteric nodes, while N4 referred to metastases in the para-aortic nodes. In the 2nd English edition, only four $\mathrm{N}$ stages were defined (N0-N3), with the N4 stage being collapsed with the N3 stage [22, 23]. However, the UICC/AJCC staging guidelines [24] consider retropancreatic, aortic, para-aortic, portal, retroperitoneal, and mesenteric metastases as M1 disease (distant metastases). In the 3rd and 4th editions of the UICC/AJCC guidelines, hepatoduodenal metastases were considered as M1 disease, while the 5th and 6th editions re-classify them as regional lymph nodes and not distant metastases [24]. The prognostic importance of lymph node involvement has been examined in many series of multivisceral resections (other than PD) for gastric cancer [6, 21, 25-28], with most finding no survival benefit for extended resections in patients with extensive lymph node involvement. However, in a few series [29], even in patients with advanced nodal disease, a benefit was found for multivisceral resection.

Though the above studies seem to imply that a highly selected group of patients could benefit from PD combined with gastrectomy, the heterogeneity of the studies included in this analysis prevents a precise identification of the indications for this aggressive multivisceral resection. The absence of staging information and the lack of information on how the decision was made to do a multivisceral resection in most studies are of great concern. Thorough pre-operative investigation will also allow for the selection of patients for neoadjuvant chemotherapy, to allow for possible down-staging, or assessment of tumor response to chemotherapy regimens. Patients who present with such extensive tumor burden that a PD is required for resection would likely benefit from the addition of chemotherapy or chemoradiation, and a multi-disciplinary approach should be explored.

This analysis is limited by the heterogeneity of the studies included. In some studies, PD was likely performed for incurable factors such as extensive nodal metastasis, or despite incurable factors such as distant metastatic disease. Additionally, the rationale for performing a simple gastrectomy was unclear in many cases, and in some series was due to incurable factors. Given the retrospective nature of all of the studies, there was likely significant selection bias for which patients were offered the PD over a simple gastrectomy. The possibility of publication bias also exists because positive outcomes will be reported more frequently. Due to the lack of quality reliable evidence, the subset of patients with locally advanced gastric cancer who may benefit from combined gastrectomy and PD could not be elucidated, and thus no indications for PD can be strongly recommended. An important endeavor will be to better define this subset of patients, as the studies reviewed present somewhat conflicting data. As surgical management of these patients continues to improve and as chemotherapeutic regimens develop, the balance of risk to benefit of this complex procedure may shift to give it an increasing role in patient management.

\section{Conclusion}

PD combined with gastrectomy is associated with a higher incidence of surgical morbidity than gastrectomy alone. There may be a subset of patients with gastric cancer invading the pancreas or duodenum in which multivisceral resection by $\mathrm{PD}$ combined with gastrectomy achieving an R0 resection may provide long-term survival in the absence of no incurable factors. However, due to the paucity of data this could not determined definitively. PD combined with gastrectomy is of no survival benefit to patients with systemic metastases or positive peritoneal disease.

Acknowledgments This research is funded by the Canadian Cancer Society (Grant \#019325). Dr. Coburn has received the Career Scientist Award through the Ontario Ministry of Health and Long-Term Care. Dr. Law is supported by the Hanna Family Chair in Surgical Oncology.

\section{References}

1. de Gara CJ, Hanson J, Hamilton S. A population-based study of tumor-node relationship, resection margins, and surgeon volume on gastric cancer survival. Am J Surg. 2003;186(1):23-7. 
2. Noguchi Y, Yoshikawa T, Tsuburaya A, Motohashi H, Karpeh MS, Brennan MF. Is gastric carcinoma different between Japan and the United States? Cancer. 2000;89(11):2237-46.

3. Maehara Y, Oiwa H, Tomisaki S, Sakaguchi Y, Watanabe A, Anai $\mathrm{H}$, et al. Prognosis and surgical treatment of gastric cancer invading the pancreas. Oncology. 2000;59(1):1-6.

4. Chan WH, Cheow PC, Chung AY, Ong HS, Koong HN, Wong WK. Pancreaticoduodenectomy for locally advanced stomach cancer: preliminary results. ANZ J Surg. 2008;78(9):767-70.

5. Martin RC 2nd, Jaques DP, Brennan MF, Karpeh M. Extended local resection for advanced gastric cancer: increased survival versus increased morbidity. Ann Surg. 2002;236(2):159-65.

6. Jeong O, Choi WY, Park YK. Appropriate selection of patients for combined organ resection in cases of gastric carcinoma invading adjacent organs. J Surg Oncol. 2009;100(2):115-20.

7. Kodama I, Takamiya H, Mizutani K, Ohta J, Aoyagi K, Kofuji K, et al. Gastrectomy with combined resection of other organs for carcinoma of the stomach with invasion to adjacent organs: clinical efficacy in a retrospective study. J Am Coll Surg. 1997; 184(1):16-22.

8. Shchepotin IB, Chorny VA, Nauta RJ, Shabahang M, Buras RR, Evans SR. Extended surgical resection in T4 gastric cancer. Am J Surg. 1998;175(2):123-6.

9. Hirose K, Onchi H, Iida A, Katayama K, Yamaguchi A, Nakagawara G. Surgical results of pancreaticoduodenectomy for carcinoma of the distal third of the stomach. Int Surg. 1999;84(1): $18-24$.

10. Saka M, Mudan SS, Katai H, Sano T, Sasako M, Maruyama K. Pancreaticoduodenectomy for advanced gastric cancer. Gastric Cancer. 2005;8(1):1-5.

11. Ajisaka H, Fujita H, Kaji M, Maeda K, Yabushita K, Konishi K, et al. Treatment of patients with gastric cancer and duodenal invasion. Int Surg. 2001;86(1):9-13.

12. Wang XB, Yang LT, Zhang ZW, Guo JM, Cheng XD. Pancreaticoduodenectomy for advanced gastric cancer with pancreaticoduodenal region involvement. World $\mathrm{J}$ Gastroenterol. 2008; 14(21):3425-9.

13. Yonemura $\mathrm{Y}$, Ooyama $\mathrm{S}$, Matumoto $\mathrm{H}$, Kamata $\mathrm{T}$, Kimura $\mathrm{H}$, Takegawa $\mathrm{S}$, et al. Pancreaticoduodenectomy in combination with right hemicolectomy for surgical treatment of advanced gastric carcinoma located in the lower half of the stomach. Int Surg. 1991;76(4):226-9.

14. Lee HJ, do Park J, Lee KU. Pancreaticoduodenectomy for locally advanced gastric cancer. Hepatogastroenterology. 2007;54(75): 977-80.

15. Menjo M, Nimura Y, Hayakawa N, Kamiya J, Kondo S, Nagino $\mathrm{M}$, et al. Ten-year survival after pancreatoduodenectomy for advanced gastric cancer-report of two cases. Hepatogastroenterology. 1999;46(26):1253-6.

16. Ozer I, Bostanci EB, Orug T, Ozogul YB, Ulas M, Ercan M, et al. Surgical outcomes and survival after multiorgan resection for locally advanced gastric cancer. Am J Surg. 2009;198(1):25-30.

17. Seevaratnam R, Cardoso R, McGregor C, Lourenco L, Mahar A, Sutradhar R, et al. How useful is pre-operative imaging for tumor, node, metastasis ( $\mathrm{T}, \mathrm{N}, \mathrm{M})$ staging of gastric cancer? A metaanalysis. Gastric Cancer. 2011.

18. Cardoso R, Coburn N, Seevaratnam R, Sutradhar R, Law C, Lourenco L, et al. A systematic review and meta-analysis on the utility of EUS for pre-operative staging for early and advanced gastric cancer. Gastric Cancer. 2011.

19. Reid-Lombardo KM, Farnell MB, Crippa S, Barnett M, Maupin $\mathrm{G}$, Bassi C, et al. Pancreatic anastomotic leakage after pancreaticoduodenectomy in 1,507 patients: a report from the Pancreatic Anastomotic Leak Study Group. J Gastrointest Surg. 2007;11(11):1451-8 (discussion 9).

20. Bassi C, Butturini G, Molinari E, Mascetta G, Salvia R, Falconi $\mathrm{M}$, et al. Pancreatic fistula rate after pancreatic resection. The importance of definitions. Dig Surg. 2004;21(1):54-9.

21. Zhang M, Zhang H, Ma Y, Zhu G, Xue Y. Prognosis and surgical treatment of gastric cancer invading adjacent organs. ANZ J Surg. 2010;80(7-8):510-4.

22. GC JRS. Japanese classification of gastric carcinoma. 1st English edition ed. Toyko: Kanehara; 1995.

23. JGCA. Japanese classification of gastric carcinoma, 2nd English edition. Gastric Cancer. 1998;1:10-24.

24. UICC/AJCC. American Joint Committee on Cancer (AJCC), Cancer Staging Manual. 1988-2002. http://www.cancerstaging. org/products/pasteditions.html.

25. Kitamura K, Tani N, Koike H, Nishida S, Ichikawa D, Taniguchi $\mathrm{H}$, et al. Combined resection of the involved organs in $\mathrm{T} 4$ gastric cancer. Hepatogastroenterology. 2000;47(36):1769-72.

26. D'Amato A, Santella S, Cristaldi M, Gentili V, Pronio ACM. The role of extended total gastrectomy in advanced gastric cancer. Hepatogastroenterology. 2004;51(56):609-12.

27. Isozaki H, Tanaka N, Tanigawa NKO. Prognostic factors in patients with advanced gastric cancer with macroscopic invasion to adjacent organs treated with radical surgery. Gastric Cancer. 2000;3:202-10.

28. Ozer I, Bostanci E, Orug T, Ozogul Y, Ulas M, Ercan M, et al. Surgical outcomes and survival after multiorgan resection for locally advanced gastric cancer. Am J Surg. 2009;198(1):25-30.

29. Saito H, Tsujitani S, Maeda Y, Fukuda K, Yamaguchi K, Ikeguchi $\mathrm{M}$, et al. Combined resection of invaded organs in patients with $\mathrm{T} 4$ gastric carcinoma. Gastric Cancer. 2001;4(4):206-11. 